\title{
Modelling the widespread effects of TOC1 signalling on the plant circadian clock and its outputs
}

\author{
Alexandra Pokhilko ${ }^{1,4}$, Paloma Mas² and Andrew J Millar ${ }^{1,3^{*}}$
}

\begin{abstract}
Background: 24-hour biological clocks are intimately connected to the cellular signalling network, which complicates the analysis of clock mechanisms. The transcriptional regulator TOC1 (TIMING OF CAB EXPRESSION 1) is a founding component of the gene circuit in the plant circadian clock. Recent results show that TOC1 suppresses transcription of multiple target genes within the clock circuit, far beyond its previously-described regulation of the morning transcription factors LHY (LATE ELONGATED HYPOCOTYL) and CCA1 (CIRCADIAN CLOCK ASSOCIATED 1). It is unclear how this pervasive effect of TOC1 affects the dynamics of the clock and its outputs. TOC1 also appears to function in a nested feedback loop that includes signalling by the plant hormone Abscisic Acid (ABA), which is upregulated by abiotic stresses, such as drought. ABA treatments both alter TOC1 levels and affect the clock's timing behaviour. Conversely, the clock rhythmically modulates physiological processes induced by ABA, such as the closing of stomata in the leaf epidermis. In order to understand the dynamics of the clock and its outputs under changing environmental conditions, the reciprocal interactions between the clock and other signalling pathways must be integrated.
\end{abstract}

Results: We extended the mathematical model of the plant clock gene circuit by incorporating the repression of multiple clock genes by TOC1, observed experimentally. The revised model more accurately matches the data on the clock's molecular profiles and timing behaviour, explaining the clock's responses in TOC1 over-expression and toc1 mutant plants. A simplified representation of ABA signalling allowed us to investigate the interactions of ABA and circadian pathways. Increased ABA levels lengthen the free-running period of the clock, consistent with the experimental data. Adding stomatal closure to the model, as a key ABA- and clock-regulated downstream process allowed to describe TOC1 effects on the rhythmic gating of stomatal closure.

Conclusions: The integrated model of the circadian clock circuit and ABA-regulated environmental sensing allowed us to explain multiple experimental observations on the timing and stomatal responses to genetic and environmental perturbations. These results crystallise a new role of TOC1 as an environmental sensor, which both affects the pace of the central oscillator and modulates the kinetics of downstream processes.

Keywords: Circadian rhythms, Biological clocks, Gene regulatory networks, Mathematical model, Systems biology

\footnotetext{
* Correspondence: andrew.millar@ed.ac.uk

'School of Biological Sciences, University of Edinburgh, Mayfield Road,

Edinburgh EH9 3JH, UK

${ }^{3}$ SynthSys, University of Edinburgh, C.H. Waddington Building, Mayfield Road,

Edinburgh EH9 3JD, UK

Full list of author information is available at the end of the article
}

\section{Biomed Central}

(c) 2013 Pokhilko et al.; licensee BioMed Central Ltd. This is an Open Access article distributed under the terms of the Creative Commons Attribution License (http://creativecommons.org/licenses/by/2.0), which permits unrestricted use, distribution, and reproduction in any medium, provided the original work is properly cited. 


\section{Background}

Circadian clocks allow most eukaryotes and some prokaryotes to anticipate the environmental day/night cycle, through rhythmic modulation of multiple physiological processes [1]. Circadian clocks are characterised by free running rhythms with a period of $\sim 24 \mathrm{~h}$ even in the absence of any environmental cues, such as in constant light conditions. Their timing is synchronised with the environmental day-night cycle principally by responses to light and temperature, but clock circuits also respond to many additional stimuli. The signalling pathways from such stimuli are often found to be rhythmically controlled by the clock, forming nested feedback loops that modify the circadian oscillator. The gene circuit of the higher plant clock consists of interlocked morning and evening loops operating through negative feedback mechanisms [2]. TOC1 is an important component of the plant clock since its mutation or overexpression dramatically change the properties of the central oscillator [3].

Although the importance of TOC1 in the plant circadian clock has long being recognized, the precise role of TOC1 in the clock has been a matter of debate [2-7]. Based on indirect genetic evidence it was first proposed that TOC1 activated the expression of the key transcription factors $L H Y$ and $C C A 1$ [4], although several observations on clock mutants remained paradoxical. Recent experimental and computational data $[2,3,7]$ have demonstrated that TOC1 functions as a repressor rather than an activator. The studies also show that TOC1 repression is not limited to CCA1 and LHY but to nearly all of the genes at the core of the oscillator [3]. The observed reduction in the expression of multiple clock genes by up-regulation of TOC1 could not be described by our previous model, where only $L H Y$ and CCA1 were affected by TOC1 [2,3]. Here, we extend our previous model by including the repression of multiple clock genes by TOC1 and explore TOC1 effect on the clock. In particular, we analysed clock dynamics in various TOC1-misexpressed lines and addressed the long-standing paradox of simultaneous reduction of LHY and CCA1 amplitudes in both toc1 mutant and TOC1-overexpressed (TOC1-ox) plants $[7,8]$.

The recently discovered pervasive negative effect of TOC1 on gene expression [3,7], the overlap between microarrays of the TOC1- and ABA-regulated genes and the gated induction of TOC1 by the stress hormone abscisic acid ABA [9] suggests that TOC1 might integrate environmental and circadian information to regulate downstream physiology at specific time of the day [9]. Here we combined the clock model with key processes regulated by $\mathrm{ABA}$ and explored mutual interactions between clock and ABA pathways. The model describes the regulation of stomatal aperture, one of the most important outputs of ABA signalling, which is gated by the clock [9]. Being a sensor of water availability in plants, ABA level increases under dry conditions, which accelerates closing of stomata in the afternoon [9-11]. In agreement with the microarray studies, TOC1 mis-expressing plants were shown to have altered ABA-dependent stomata closure and affected responses to drought conditions [9]. To model the observed gating of stomatal opening by the clock, we included the interaction of ABA with the ABAR protein (also known as the $\mathrm{H}$ subunit of $\mathrm{Mg}$ chelatase, CHLH or GUN5) - a key component of the ABA signalling pathway, arguably postulated as ABA receptor [12]. Besides being one of the downstream targets of TOC1, ABAR was shown to be a key component in the gating of ABA signalling by the clock $[9,13]$. We used the model to explore the dynamics of the opening of stomatal pore under various genetic and environmental perturbations, such as mutations of clock genes and by changing the light and water conditions, and showed that the model provides good match to existing experimental data. Our results show how the dual roles of TOC1 are integrated, combining timing and environmental information to modulate downstream physiology.

\section{Results and discussion \\ Model description}

The latest plant clock model [2], herein called P2011, was revised by adding the recently discovered negative regulation of the oscillator genes by TOC1 (blue lines on Figure 1A) as described in the section 1 below. Additionally, we have included a simplified version of the main steps leading to the induction of TOC 1 by $\mathrm{ABA}$ and the regulation of stomata aperture (Figure 1B), described in the section 2 below.

1. Extending the clock model by inhibition of gene expression by TOC1

The latest model of the Arabidopsis oscillator [2] was extended based on our recent data on the inhibition of expression of multiple clock genes by TOC1 and induction of TOC1 through ABA signalling. The model is described by a system of 32 ODEs. The basic structure of the plant clock was kept from our previous P2011 model [2]. Briefly, the key components of the clock are the $L H Y$ and CCA1 morning genes and the evening complex (EC) genes. $L H Y$ and CCA1 are similarly expressed around dawn and act together to regulate multiple targets. They are described by single variable $L H Y / C C A 1$ as before. EC (EVENING COMPLEX) genes LUX, ELF3 (EARLY FLOWERING 3) and ELF4 are expressed around dusk and form the EC protein complex, which suppresses expression of multiple 


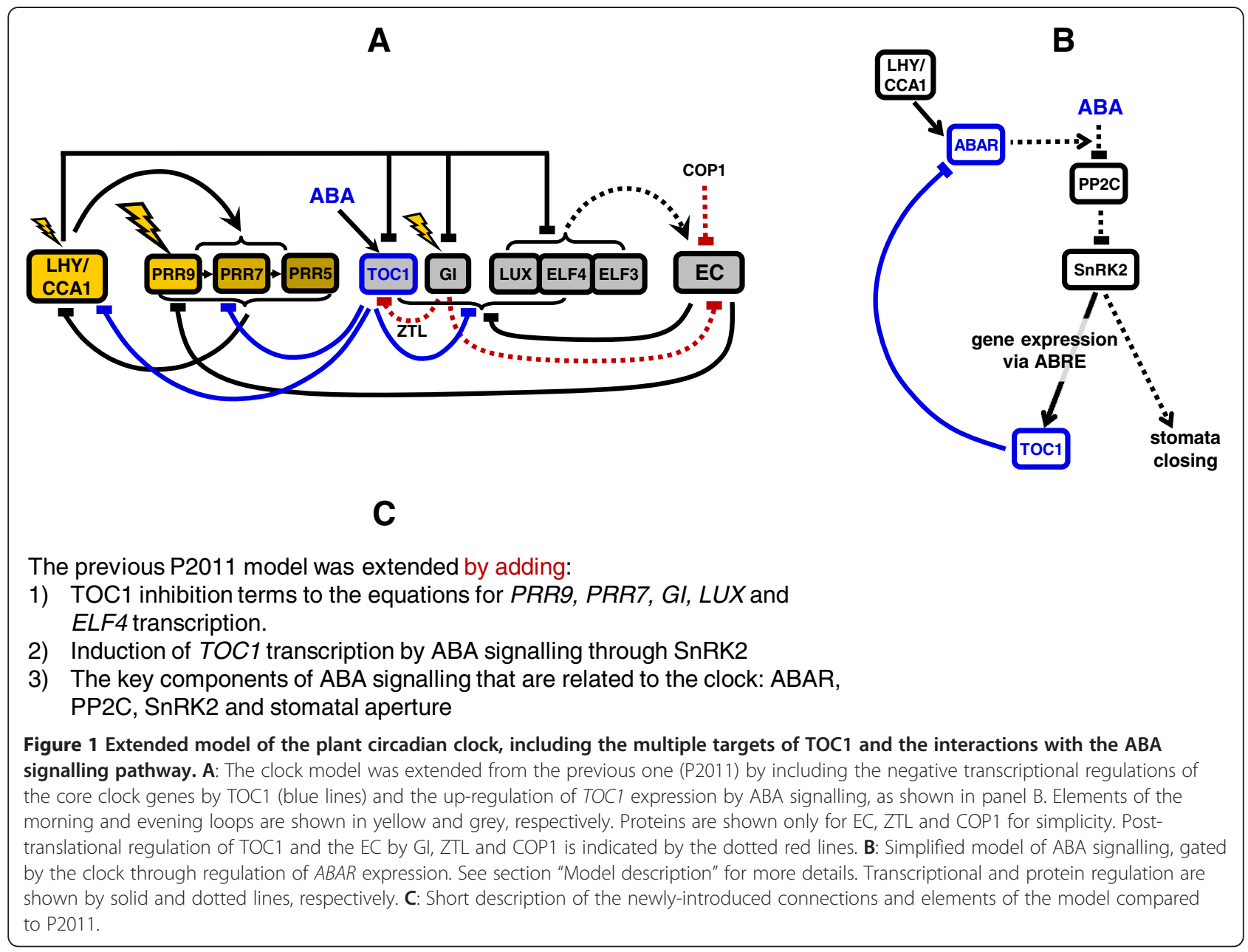

genes at night (Figure 1; [2]). LHY/CCA1 expression is suppressed by the PRR proteins in a morning loop, while the EC is negatively regulated by the ubiquitin E3 ligase COP1 (CONSTITUTIVE PHOTOMORPHOGENIC 1), which targets ELF3 protein to degradation by proteasome and by GI protein, in the evening loop. The morning and evening loops are further interlocked through the suppression of evening gene expression by $L H Y / C C A 1$ and suppression of PRR9 and TOC1 (PRR1) expression by the EC. The model also includes the $F$ box protein ZTL (ZEITLUPE), which negatively regulates the level of TOC1 protein. Light entrains the clock similarly to the P2011 model through several mechanisms, which are supported by experimental data [2]. The main mechanisms include acute activation of $L H Y / C C A 1, P R R 9$ and GI transcription (eqs. 1, 5, 27) immediately after dawn; stabilization of PRR proteins in presence of light (eqs. 6, 8, 10) and light-dependent regulation of the EC by COP1 and GI proteins (eqs. 24, 21, 22).
The TOC1-related reactions were substantially extended. Firstly, we added multiple reactions of inhibition of clock gene expression by TOC1. This includes direct inhibition of expression of LHY/CCA1 as in the P2011 model (eq. 1), and also PRR9, PRR7, PRR5, LUX, ELF4 and GI (eqs. $5,7,9,18,14,27)$, which is based on our data [3] (Figure 1A). The details of TOC1 interactions with other regulators (e.g. LHY/CCA1, EC, protein $\mathrm{P}$ ) to modulate gene expression are largely unknown, so we assumed that TOC1 acts as a non-competitive inhibitor of gene expression Secondly, we added physiologically relevant activation of TOC1 expression by the ABA signalling pathway (Figure 1B, [9]), described in section 2 below. This provided an additional level of input to the clock through ABA, which is induced by stress. Thirdly, we added regulation of stomata aperture as a clock output, which is directly related to both ABA and TOC1 signalling (Figure 1B), as presented in section 2 below. 
The model is described by the following system of ODEs:

$$
\begin{aligned}
\frac{d c_{L}^{m}}{d t}= & q_{1} L c_{P} \\
& +n_{1} \frac{g_{1}{ }^{a}}{g_{1}{ }^{a}+\left(c_{P 9}+c_{P 7}+c_{P 5}+c_{T}\right)^{a}} \\
& -\left(m_{1} L+m_{2} D\right) \cdot c_{L}^{m} \\
\frac{d c_{L}}{d t}= & \left(p_{2}+p_{1} L\right) \cdot c_{L}^{m}-m_{3} c_{L}-p_{3} \frac{c_{L}{ }^{c}}{c_{L}{ }^{c}+g_{3}{ }^{c}} \\
\frac{d c_{L \bmod }}{d t}= & p_{3} \frac{c_{L}{ }^{c}}{c_{L}{ }^{c}+g_{3}{ }^{c}}-m_{4} c_{L \bmod } \\
\frac{d c_{P}}{d t}= & p_{7} D \cdot\left(1-c_{P}\right)-m_{11} c_{P} L \\
\frac{d c_{P 9}^{m}}{d t}= & \frac{g_{18}{ }^{g}}{g_{18}{ }^{g}+c_{T}^{g}} \\
& \times\left(L \cdot q_{3} \cdot c_{P}+\frac{g_{8}}{g_{8}+c_{E C}}\left(n_{4}+n_{7} \cdot \frac{c_{L}{ }^{e}}{g_{9}{ }^{e}+c_{L}{ }^{e}}\right)\right) \\
& -m_{12} c_{P 9}^{m}
\end{aligned}
$$

$$
\frac{d c_{P 9}}{d t}=p_{8} c_{P 9}^{m}-\left(m_{13}+m_{22} D\right) \cdot c_{P 9}
$$

$$
\begin{aligned}
\frac{d c_{P 7}^{m}}{d t}= & \frac{g_{22}{ }^{g}}{g_{22}{ }^{g}+c_{T}{ }^{g}} \\
& \times\left(n_{8} \frac{c_{\text {Ltot }}^{e}}{g_{10^{e}}{ }^{e}+c_{L t o t}{ }^{e}}+n_{9} \frac{c_{P 9}{ }^{f}}{g_{11}^{f}+c_{P 9}^{f}}\right) \\
& -m_{14} c_{P 7}^{m}
\end{aligned}
$$

$$
\frac{d c_{P 7}}{d t}=p_{9} c_{P 7}^{m}-\left(m_{15}+m_{23} D\right) \cdot c_{P 7}
$$

$$
\begin{aligned}
\frac{d c_{P 5}^{m}}{d t}= & \frac{g_{23}{ }^{g}}{g_{23}{ }^{g}+c_{T}{ }^{g}} \\
& \times\left(n_{10} \frac{c_{L \bmod }{ }^{e}}{g_{12}{ }^{e}+c_{L \bmod }}+n_{11} \frac{c_{P 7}^{b}}{g_{13} b+c_{P 7}^{b}}\right) \\
& -m_{16} c_{P 5}^{m}
\end{aligned}
$$

$$
\begin{aligned}
\frac{d c_{P 5}}{d t}= & p_{10} c_{P 5}^{m}-\left(m_{17}+m_{24} D\right) \cdot c_{P 5} \\
\frac{d c_{T}^{m}}{d t}= & \frac{n_{2}}{1+\left(c_{L} /\left(g_{5} \cdot\left(1+\left(c_{S n R K 2} / g_{25}\right)^{j}\right)\right)\right)^{e}} \\
& \cdot \frac{g_{4}}{g_{4}+c_{E C}}-m_{5} c_{T}^{m}
\end{aligned}
$$

$$
\begin{aligned}
\frac{d c_{T}}{d t}= & p_{4}\left(c_{T}^{m}+n_{16}\right) \\
& -\left(m_{6}+m_{7} D\right) \cdot c_{T}\left(c_{Z T L} \cdot p_{5}+c_{Z G}\right) \\
& -m_{8} c_{T}
\end{aligned}
$$

$$
\begin{aligned}
\frac{d c_{E 4}^{m}}{d t}= & n_{15} \cdot \frac{g_{21}{ }^{g}}{g_{21}^{g}+c_{T}^{g}} \cdot \frac{g_{20}}{g_{20}+c_{E C}} \cdot \frac{g_{6}{ }^{e}}{g_{6}{ }^{e}+c_{L}{ }^{e}} \\
& -m_{34} c_{E 4}^{m}
\end{aligned}
$$

$$
\frac{d c_{E 4}}{d t}=p_{23} c_{E 4}^{m}-m_{35} c_{E 4}-p_{25} c_{E 4} c_{E 3 n}
$$$$
+p_{21} c_{E 34}
$$

$\frac{d c_{E 3}^{m}}{d t}=n_{3} \frac{g_{16}{ }^{e}}{g_{16^{e}}{ }^{e}+c_{L}{ }^{e}}-m_{26} c_{E 3}^{m}$

$\begin{aligned} \frac{d c_{E 3 c}}{d t}= & p_{16} c_{E 3}^{m}-m_{9} c_{E 3 c} c_{C O P 1 c}-p_{17} c_{E 3 c} c_{G c} \\ & -p_{19} c_{E 3 c}+p_{20} c_{E 3 n}\end{aligned}$

$$
\begin{aligned}
\frac{d c_{E 3 n}}{d t}= & p_{19} c_{E 3 c}-p_{20} c_{E 3 n}-p_{17} c_{E 3 n} c_{G n} \\
& -m_{9} c_{E 3 n} \cdot c_{C O P 1 d}-m_{10} c_{E 3 n} \cdot c_{C O P 1 n} \\
& +p_{21} c_{E 34}-p_{25} c_{E 4} c_{E 3 n}
\end{aligned}
$$

$$
\begin{aligned}
\frac{d c_{L U X}^{m}}{d t}= & n_{13} \cdot \frac{g_{19^{g}}}{g_{19^{g}}+c_{T}{ }^{g}} \cdot \frac{g_{2}}{g_{2}+c_{E C}} \cdot \frac{g_{6}{ }^{e}}{g_{6}{ }^{e}+c_{L}{ }^{e}} \\
& -m_{34} c_{L U X}^{m}
\end{aligned}
$$

$$
\frac{d c_{L U X}}{d t}=p_{27} c_{L U X}^{m}-m_{36} c_{L U X}-p_{26} c_{L U X} c_{E 34}
$$

$\frac{d c_{C O P 1 c}}{d t}=n_{5}-p_{6} c_{C O P 1 c}$

$$
-m_{27} c_{C O P 1 c}\left(1+p_{15} L\right)
$$

$\frac{d c_{C O P 1 n}}{d t}=p_{6} c_{C O P 1 c}-n_{6} L \cdot c_{P} \cdot c_{C O P 1 n}$

$$
\begin{aligned}
& -n_{14} c_{C O P 1 n} \\
& -m_{27} c_{C O P 1 n}\left(1+p_{15} L\right)
\end{aligned}
$$

$$
\begin{aligned}
\frac{d c_{C O P 1 d}}{d t}= & n_{14} c_{C O P 1 n}+n_{6} L \cdot c_{P} \cdot c_{C O P 1 n} \\
& -m_{31}\left(1+m_{33} D\right) \cdot c_{C O P 1 d}
\end{aligned}
$$

$$
\begin{aligned}
\frac{d c_{E G c}}{d t}= & p_{17} c_{E 3 c} c_{G c}-m_{10} c_{E G c} c_{C O P 1 c} \\
& -p_{18} c_{E G c}+p_{31} c_{E G n}
\end{aligned}
$$

$$
\begin{aligned}
\frac{d c_{E C}}{d t}= & p_{26} c_{L U X} c_{E 34}-m_{10} c_{E C} \cdot c_{C O P 1 n} \\
& -m_{9} c_{E C} \cdot c_{C O P 1 d} \\
& -m_{32} c_{E C}\left(1+p_{24} \cdot L \cdot \frac{c_{G n_{t o t}}{ }^{d}}{g_{7}{ }^{d}+c_{G n_{\text {tot }}} d}\right)
\end{aligned}
$$




$$
\begin{aligned}
\frac{d c_{Z T L}}{d t}= & p_{14}-p_{12} L c_{Z T L} c_{G c}+p_{13} c_{Z G} D \\
& -m_{20} c_{Z T L} \\
\frac{d c_{Z G}}{d t}= & p_{12} L c_{Z T L} c_{G c}-p_{13} c_{Z G} D-m_{21} c_{Z G} \\
\frac{d c_{G}^{m}}{d t}= & \frac{g_{17}{ }^{g}}{g_{17^{g}}+c_{T}^{g}} \cdot \\
& \times\left(L \cdot q_{2} \cdot c_{P}+n_{12} \frac{g_{14}}{g_{14}+c_{E C}} \cdot \frac{g_{15}{ }^{e}}{g_{15}{ }^{e}+c_{L}}\right) \\
& -m_{18} c_{G}^{m}
\end{aligned}
$$

$$
\begin{aligned}
\frac{d c_{G c}}{d t}= & p_{11} c_{G}^{m}-p_{12} L c_{Z T L} c_{G c}+p_{13} c_{Z G} D \\
& -m_{19} c_{G c}-p_{17} c_{E 3 c} c_{G c}-p_{28} c_{G c} \\
& +p_{29} c_{G n}
\end{aligned}
$$

$$
\begin{aligned}
\frac{d c_{A B A R}^{m}}{d t}= & n_{17} \cdot \frac{g_{24}{ }^{g}}{g_{24^{g}}+c_{T^{g}}} \cdot \frac{c_{L}^{e}}{g_{28}{ }^{e}+c_{L}^{e}} \\
& -m_{37} c_{A B A R}^{m} \\
\frac{d c_{P P 2 C}}{d t}= & p_{33} \cdot \frac{g_{27}{ }^{h}}{g_{27^{h}+c_{A R}{ }^{h}}}-m_{39} c_{P P 2 C}
\end{aligned}
$$

$$
\frac{d c_{\text {SnRK } 2}}{d t}=p_{32}-m_{30} \cdot c_{S n R K 2} \cdot c_{P P 2 C}
$$

$$
\begin{aligned}
\frac{d s}{d t}= & \left(n_{19}+n_{18} L\right) \cdot \frac{g_{26}^{i}}{g_{26}^{i}+c_{S n R K 2}^{i}} \cdot(1-s) \\
& -m_{29} s
\end{aligned}
$$

$$
\begin{aligned}
c_{E 34}= & p_{25} c_{E 4} c_{E 3 n} /\left(p_{26} c_{L U X}+p_{21}+m_{9} c_{C O P 1 d}\right. \\
& \left.+m_{10} c_{C O P 1 n}\right)
\end{aligned}
$$

$$
c_{E G n}=\left(p_{18} c_{E G c}+p_{17} c_{E 3 n} c_{G n}\right) /\left(m_{10} c_{C O P 1 n}\right.
$$$$
\left.+m_{9} c_{C O P 1 d}+p_{31}\right)
$$$$
c_{G n}=p_{28} c_{G c} /\left(p_{29}+m_{19}+p_{17} c_{E n}\right)
$$$$
c_{G n_{t o t}}=c_{G n}+c_{E G n}
$$$$
c_{A R}=0.5 \cdot\left(A_{0}+c_{A B A R}^{m}+g_{29}\right.
$$$$
\left.-\sqrt{\left(A_{0}+c_{A B A R}^{m}+g_{29}\right)-4 A_{0} c_{A B A R}^{m}}\right)
$$

Where $c_{i}^{m}$ and $c_{i}$ stand for dimensionless concentrations of mRNA and protein, respectively. The time unit is an hour. Index " $i$ " labels the molecular components as follows:

ABAR ABAR mRNA

$A R$ ABA complex with ABAR protein
COP1c COP1 cytoplasmic protein COP1d COP1 nuclear protein, day COP1n COP1 nuclear protein, night E3 ELF3 mRNA

E3c ELF3 cytoplasmic protein

E3n ELF3 nuclear protein

E4 ELF4 mRNA and protein

E34 nuclear protein complex ELF3-ELF4

$E C$ nuclear protein complex ELF4-ELF3-LUX

$E G c$ cytoplasmic protein complex ELF3-GI

$E G n$ nuclear protein complex ELF3-GI

$G$ GI mRNA

Gc GI cytoplasmic protein

Gn GI nuclear protein

Gn_tot total amount of GI protein in nucleus

$L$ LHY/CCA1 mRNA and protein

Lmod LHY/CCA1 modified protein

Ltot total amount of LHY/CCA1 protein

LUX LUX mRNA and protein

P5 PRR5 mRNA and protein

P7 PRR7 mRNA and protein

P9 PRR9 mRNA and protein

$P P 2 C$ PP2C active protein

SnRK2 SnRK2 active protein

$T$ TOC1 mRNA and protein

ZG cytoplasmic protein complex GI-ZTL

ZTL ZTL protein

Symbol "s" corresponds to stomata aperture in relative units (the maximum of $s$ is equal to 1 ). $A_{0}$ is the total ABA level in relative units. ABA level for most of calculations was set to 1 , unless it is stated otherwise $\left(\mathrm{ABA}_{0}=1\right)$.

The quasi-steady state approximation for the nuclear complexes ELF3-GI, ELF3-ELF4 and nuclear GI protein, which reduces the number of equations, was taken from the P2011 model. COP1 nuclear activity was described by two forms of COP1 ([2], eqs. 20, 21). The parameters $n_{j}$ represent the rate constants of transcription and stomata opening, $m_{j}$ are the rate constants of degradation; $p_{j}$ are constants of translation, protein modification and protein complex formation; $g_{j}$ are Michaelis-Menten constants and $a, b, c, d, e, f, g, h, i, j$ are Hill coefficients; $q_{j}$ are the rate constants of acute (P-dependent) light activation of transcription. The acute light response in activation of $P R R 9, L H Y / C C A 1, G I$, transcription (eqs. $5,1,27$ ) was modelled using a light-sensitive activator - protein $\mathrm{P}\left(c_{P}\right)$, which is accumulated in darkness and was degraded in light similarly to the P2011 model (eq. 4). $\mathrm{L}=1$ when light is present, 0 otherwise; $\mathrm{D}=1-\mathrm{L}$. The $L$ $(t)$ function was used to simulate smooth 
transitions between $\mathrm{L}$ and $\mathrm{D}$ analogous to the P2011 model:

$$
\begin{aligned}
L(t)= & 0.5 \cdot((1+\tanh ((t-24 \cdot \text { floor }(t / 24)-\text { dawn }) / T)) \\
& -(1+\tanh ((t-24 \cdot \text { floor }(t / 24)-\text { dusk }) / T)) \\
& +(1+\tanh ((t-24 \cdot \text { floor }(t / 24)-24) / T)))
\end{aligned}
$$

Where dawn and dusk are the phases of dawn and dusk (normally dawn $=0$ ); $\mathrm{T}$ is the duration of twilight (we used $\mathrm{T}=0.05 \mathrm{~h}$ ); tanh and floor standard functions of hyperbolic tangent and rounding operation. This representation of periodic input corresponds to the Input Signal Step Function, which now has convenient software support in SBML [14]. Mutations of the clock genes were simulated by decreasing the rate of transcription of the corresponding gene to zero. TOC1-ox plants were modelled by adding the background activation of TOC1 translation (parameter n16 in eq. 12; n16 = 0 for wild type). The equations for COP1 E3 ligase activities (eqs. 20, 21), which were developed in P2011, are independent of the clock and their parameters were constrained from the data on the kinetics of COP1 substrates HFR1 and HY5 proteins upon dark/light transitions similarly to P2011 [15]. The equations for HY5 and HFR1 proteins, which were used only for the optimization of COP1 parameters, are taken from P2011:

$$
\begin{aligned}
d c_{H Y 5} / d t= & p_{22}-m_{38} c_{H Y 5} c_{C O P 1 d} \\
& -m_{25} c_{H Y 5} c_{C O P 1 n} \\
d c_{H F R 1} / d t= & p_{30}-m_{28} c_{H F R 1} c_{C O P 1 n}
\end{aligned}
$$

2. Modelling of ABA signalling and its relation with the clock

To model the mutual connections between the clock and ABA signalling, we extended the clock model by including $\mathrm{ABA}$-induced reactions.

The first group of reactions of ABA signalling is related with the inactivation of protein phosphatase PP2C (protein phosphatase of $2 \mathrm{C}$ type) by the ABA complex ([11]; Figure 1B). Based on the data showing relatively small diurnal changes in ABA concentration in plants $[10,16]$, we assumed that ABA level is constant in our model and treated it as a parameter $\mathrm{ABA}_{0}$. Next we included the potential ABA receptor ABAR, which is a necessary component of the regulation of both TOC1 expression and stomatal aperture by ABA [9]. $A B A R$ expression has a strong circadian pattern, peaking in the morning [9]. Our analysis of the $A B A R$ promoter in Arabidopsis revealed 2 CCA1 binding sites (CBS) AAATCT [17], in addition to the known
TOC1-binding sites [3,9], suggesting a possible mechanism for the regulation of $A B A R$ transcription by the clock. This was described in our model through activation of $A B A R$ expression by LHY/ CCA1 and its inhibition by TOC1 ([3,17], eq. 29). To simplify the model and reduce the number of equations, we used a quasi-steady state approximation for the ABA complex with ABAR, assuming that ABAR protein level follows the cognate mRNA. PP2C activity was described through its inhibition by this ABA complex (eq. 30). The second important group of reactions of ABA signalling is related with the activation of SNF1 (Sucrose-Nonfermenting Kinase1)-related protein kinase SnRK2 by ABA [11]. This activation happens through double-negative interactions: the ABA complex inactivates $\mathrm{PP} 2 \mathrm{C}$ and $\mathrm{PP} 2 \mathrm{C}$ inactivates SnRK2. So we included in the model an equation for SnRK2 activity, which is inhibited by PP2C (eq. 31). After activation by ABA, SnRK2 mediates multiple reactions of the $\mathrm{ABA}$ pathway, including induction of transcription of multiple target genes which possess ABRE elements (ABA-responsive elements) in their promoters [11]. As TOC1 was reported to be induced by ABA [9], we analysed the TOC1 promoter and identified 2 ABRE elements. This suggested that a mechanism of TOC1 induction by ABA is similar to other genes and mediated by SnRK2 (Figure 1B). Therefore we included in the model the activation of TOC1 expression by SnRK2, assuming that this activation competes with inhibition of TOC1 by LHY/CCA1 protein ([9], eq. 11). An analogous mechanism of ABA signalling through the ABA/PP2C/SnRK2 pathway is involved in the acceleration of stomatal closing by ABA [11], which we included into the model. The equation for stomatal aperture describes stomatal opening in a phenomenological way, which allowed us qualitatively compare the model behaviour with physiological data. Additionally to the inhibition of stomatal opening by ABA signalling through active SnRK2, we included the known activation of stomatal opening in the presence of light ([18], eq. 32). So finally the clock model was extended by including eqs. (29)-(32) for $A B A R, \mathrm{PP} 2 \mathrm{C}, \mathrm{SnRK} 2$ and stomata aperture (variable "s"). Thus we connected the clock with a simple version of ABA signalling, which enabled us to simulate TOC1 and stomatal regulation by $A B A$. The effective parameters of the ABA-related reactions were optimized together with other parameters the clock model against multiple perturbations as described below.

Additional file 1: Figure S1 shows simulated diurnal kinetics of the main components of ABA signalling 
under the optimal parameters, which are presented in Additional file 2: Table S1. The peak of $A B A R$ transcription soon after dawn corresponds to the publicly available microarray data (http://diurnal. mocklerlab.org/), which agrees with RT-Q-PCR analysis [9]. The accumulation of ABA-ABAR complex in the day time leads to inactivation of PP2C and activation of SnRK2, with the peak of SnRK2 activity in the afternoon (Additional file 1: Figure S1). SnRK2 then induces TOC1 expression and stimulates the closing of stomata as described above.

3. Solving the system of ODEs

The extension of P2011 by adding newly discovered processes of the inhibition of the PRR9, PRR7, PRR5, LUX, ELF4 and GI clock genes by TOC1 (Figure 1A) resulted in the addition of 7 new parameters to P2011. Our model was also extended compared to P2011 by adding reactions describing ABA-related processes (Figure 1B), which introduced 14 more new parameters. Finally, the model describes the dynamics of stomatal opening, which was absent from P2011, resulting in 5 more new parameters. Therefore, the higher level of biological complexity of the model and its extension to hormonal signalling through ABA inevitably increased the parameter space, adding 26 parameters compared to P2011. This allowed our model to describe experimental data that were not described by P2011, such as the inhibition of multiple clock genes by TOC1, stimulation of TOC1 expression by ABA signalling, changing the clock period by ABA and the dynamics of stomata presented in the Results below. In addition, the new structure of the clock, which now incorporates ABA signalling to the clock through TOC1 (Figure 1A) allowed us to improve the description of the data on the clock kinetics compared to P2011, as shown in the Results below. Parameter values were either constrained based on experimental data or fitted to multiple time-series data sets, similarly to the P2011 model. 49 out of the total 133 parameters were constrained based on the available experimental data. Most of these parameters have the same values as in P2011 because they were constrained by the same data. Other parameters were fitted to multiple datasets similarly to P2011, but their values may be different from P2011 because the structures of the models are different. The optimal set of parameters is presented in Additional file 2: Table S1. The new model, under the optimal parameter values, retains most of its properties from P2011, keeping the good fit to data from various conditions, such as diurnal cycles of the wild type and lhy/ccal, lhy/ccal/gi and elf3 mutants, as well as continuous light or darkness in the wild type and multiple mutants. For example, the period values for the simulated free-running clock in wild type and mutant plants were $24.5 \mathrm{~h}$ for wild type under constant light conditions, $26.6 \mathrm{~h}$ for wild type under constant darkness and $17.7 \mathrm{~h}$, 21.4 h, 30.1 h, 30.7 h, 21.2 h for lhy/cca1, toc1, ztl, prr79 and gi mutants in constant light, which match the experimental observations [8,19-22]. Additionally lhy/ccal/gi, elf3, elf4 and lux mutants were arrhythmic in constant light, in agreement with the data [8,23-25]. We also tested the sensitivity of the model to the variations of the newly introduced parameters of the inhibition of target genes by TOC1 and ABA signalling. Additional file 1: Figure S2 shows the relative changes in the amplitude of $L H Y$ expression and the clock period in constant light conditions under $10 \%$ changes of each parameter. We observed less than $3 \%$ changes in the period and less than $12 \%$ change in the amplitude of $L H Y$ mRNA under variations of these parameters, which shows that the extended model retains its robustness to parameter perturbations from P2011 (Additional file 1: Figure S2). Thus, the optimal set of parameters demonstrated good correspondence to multiple datasets and provides robust behaviour in the model. However, given the significant complexity of the model, which is related to the complexity of the biological processes involved, and the number of unknown parameters, we cannot exclude the existence of other parameter sets that might equally describe the data. The detailed study of this large parameter space lies outside the scope of this paper. The model with the current parameter set matches well enough to the data to make it a useful instrument for the exploration of the possible mechanisms of the observed biological phenomenon. ABA modulates expression of the clock genes through the induction of TOC1 expression followed by suppression of TOC 1 target genes. Additional file 1: Figure S3 shows the dependence of the peak mRNA values of all clock genes on the ABA levels, demonstrating that ELF4, LUX, GI, PRR7 and PRR5 are the most sensitive to ABA in our model.

\section{Modulation of circadian dynamics via clock gene repression by TOC1}

The extension of our previous P2011 model with the parallel suppressive effects of TOC1 on the oscillator genes (Figure 1A; [3]) allowed us to improve the description of data from several clock mutants compared to P2011, without affecting the good fit of the P2011 model to the data of other mutants. Figure 2 shows the profiles of clock gene 
A

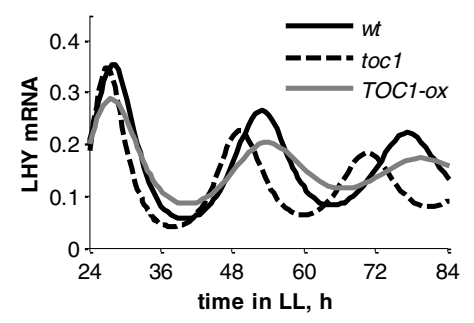

\section{B}

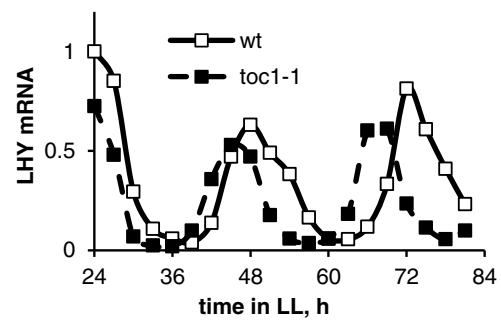

C
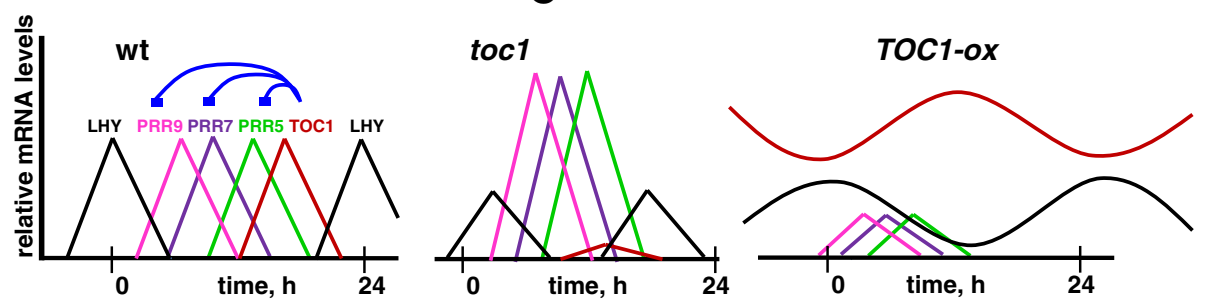

Figure $\mathbf{2}$ Clock gene expression profiles in TOC1 mis-expressed lines. A: The simulated profiles of LHY/CCA1 mRNA under constant light conditions are shown for wild type (wt), toc1 and TOC1-ox lines by black solid, dashed and grey lines, respectively. TOC1-ox was simulated with $\mathrm{n} 16=0.1$. Simulations were run after entrainment of the clock to $12 \mathrm{~L}: 12 \mathrm{D}$ conditions to match the data. $\mathbf{B}$ : Experimental data for $L H Y$ mRNA in wild type and toc1 plants under constant light conditions are redrawn from [4]; C: Cartoon illustrating the clock gene profiles in TOC1 misexpressed lines relative to the wild type. Coloured lines represent the expression of $L H Y$ and its inhibitors PRR9, PRR7, PRR5, TOC1, as shown in the legend for the wild type. Blue connections on the wild-type panel (left) show the inhibition of PRR9, PRR7, PRR5 by TOC1. The release of this inhibition in the tocl mutant (centre) results in the increase in PRR expression, which causes the reduction of the LHY amplitude and, together with the absence of TOC1, shortens the clock period in the tocl mutant. On the contrary, overexpression of TOC1 (right) reduces PRR expression and allows $L H Y$ levels to rise in the morning, while higher TOC1 delays LHY at the end of the night, lengthening the period in TOC1-OX.

expression in the simulated TOC1-mis-expressing plants and explains the mechanisms involved. The observed decrease in the peak level of $L H Y / C C A 1$ expression in the toc1 mutant suggested that TOC1 was an activator of $L H Y$ and CCA1 [4]. Although TOC1 is a repressor of $L H Y / C C A 1$ in the model, removing TOC1 also reduces $L H Y / C C A 1$ expression (Figure 2A). The simulated toc1 mutation increases the levels of the remaining $L H Y / C C A 1$ inhibitors, the PRR (PSEUDO-RESPONSE REGULATOR) proteins (PRR9, PRR7, PRR5), by relieving the negative regulation of $P R R$ transcription by TOC1 (Figure $2 \mathrm{C}$ ). In the model, this results in instant down-regulation of $L H Y$ / CCA1 mRNA after switching from a simulated light/dark cycle to constant light (LL) conditions. Figure 2A demonstrates that the simulated toc 1 mutant has $L H Y / C C A 1$ at 0.85 -fold the peak level in wild type after two days in LL, which is close to the experimentally observed 0.82 -fold for $L H Y$ mRNA (Figure 2B, $[4,26])$. In contrast, the toc 1 mutant simulated in the P2011 model initially contradicts the data, as it first shows an increase of $L H Y / C C A 1$ levels followed by only a slight decrease ( 0.97 -fold after two days of LL), and through a more indirect mechanism [2].

Over-expression of TOC1 also resulted in a lower level of $L H Y / C C A 1$ expression, which is due to the direct suppression of $L H Y / C C A 1$ expression by the increased level of the repressor, TOC1 (Figure 2A). This effect of TOC1 was present already in the P2011 model. However, the
P2011 model failed to describe the longer period of the TOC1-overexpressor line (TOC1-ox) under constant light, which was observed in the data [27]. The discrepancy in the earlier model was caused by increased levels of the PRR inhibitors as well as TOC1, which resulted in a reduction of the $L H Y / C C A 1$ level. This led to a shortening of the period in the simulated TOC1-ox lines in P2011 rather than a longer period. The current model describes the experimentally observed prolongation of the period due to delay in LHY/CCA1 in TOC1-ox compared to the wild type because repression by TOC1 reduces the level of the other PRRs (Figure 2C). The simulated TOC1-ox in Figure $2 \mathrm{~A}$ has a $1.5 \mathrm{~h}$ longer period than the wild type. The period difference between the TOC1-ox and wild type increases with the increase in the level of TOC1 expression in our model, in agreement with the data [21,27]. For example, 3-fold increase of the TOC1 overexpression level compared to the level shown in Figure 2A results in $5 \mathrm{~h}$ longer period of the TOC1-ox compared the wild type (while it has $1 \mathrm{~h}$ shorter period in P2011). The large prolongation of the TOC1-ox period ( $5 \mathrm{~h}$ ) corresponds to the experimental observations for the TOC1-ox lines with high overexpression levels of TOC1 [27]. The oscillations are damping in TOC1-ox in our model simulations (Figure 2A) similarly to the data [27], to the point of the loss of rhythmicity of the simulated TOC1-ox at very high levels of TOC1-overexpression, which also agrees with the 

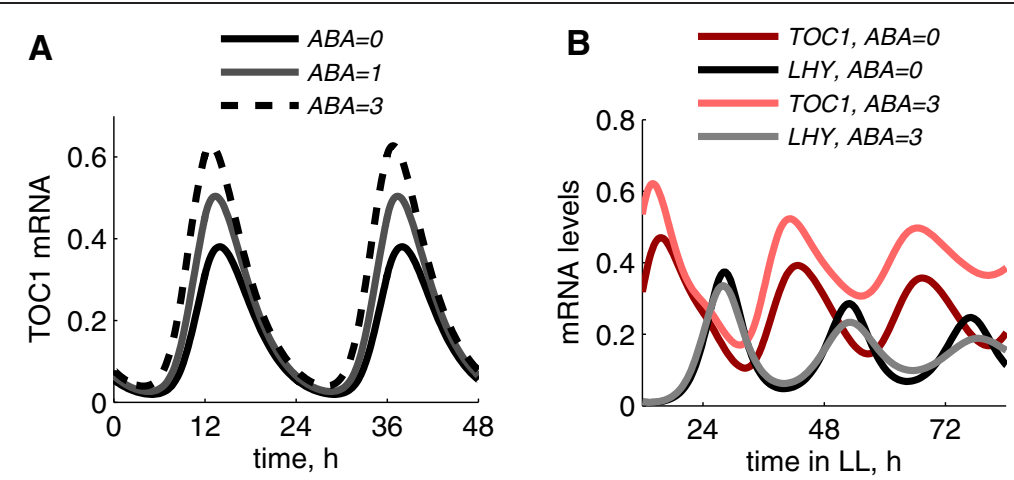

Figure 3 Modulation of clock dynamics by ABA. Diurnal profiles of TOC1 mRNA (A) under various levels of ABA (ABA level is expressed in relative units; the wild-type level is 1). Simulations were run under a 12 L:12D diel cycle. B: Free running oscillation of $L H Y / C C A 1$ and TOC1 mRNA under constant light conditions in the absence or presence of ABA. The $1.3 \mathrm{~h}$ period lengthening in the presence of increased ABA in the model simulation is close to the reported $1.5 \mathrm{~h}$ lengthening of the period of the clock marker CAB2: LUC under constant light conditions [29].

data [21]. The model suggests that the period increase is due to the simultaneous up-regulation of TOC1 and down-regulation of the other $P R R$ expression in the TOC1-ox lines, which allows a longer interval of $L H Y /$ CCA1 expression than in the wild type.

Figure $2 \mathrm{C}$ shows schematically the mechanisms of regulation of $L H Y / C C A 1$ expression by the wave of PRR inhibitors in lines with different levels of TOC1: In the toc1 mutant, increased PRR levels reduce $L H Y / C C A 1$ levels, while the absence of TOC1, the last inhibitor, shortens the period. In TOC1-ox, LHY/CCA1 expression is repressed mainly by TOC 1 protein, which declines when $L H Y$ is raising (LHY and TOC1 are anti-phasic) and thus allowing oscillations to occur but with longer period than in wild type (Figure 2A). Thus the model offers an explanation of the low $L H Y$ and CCA1 levels in the toc1 mutant [4] that is consistent with the biochemical data showing that TOC1 is a repressor [3,7]. The mechanism depends upon the inhibition of multiple clock genes by TOC1, particularly the PRRs (Figure 2C).

Additionally to affecting the expression of the morning genes $L H Y / C C A 1, P R R 9, P R R 7, P R R 5$, TOC1 negatively regulates the evening genes $L U X$ (LUX ARRHYTHMO), ELF4 (EARLY FLOWERING 4) and GI (GIGANTEA) in the experimental data [3] and in our model. This is in clear contrast with the P2011 model, where TOC1 affected only $L H Y / C C A 1$ expression directly. The new model suggests that increased levels of evening gene expression result in the arrhythmia of the $/ \mathrm{hy} / \mathrm{ccal} / \mathrm{tocl}$ triple mutant under constant light conditions, as opposed to the damped oscillation of the lhy/ccal double mutant. This agrees with experimental observations [28].

\section{TOC1-mediated effects of $A B A$ on circadian timing}

Figure $3 \mathrm{~A}$ shows simulated diurnal profiles of TOC1 expression under increasing $\mathrm{ABA}$ levels, which might correspond to increasing levels of stress, such as drought
[9,10]. In agreement with the data [9], ABA accelerates TOC1 expression in the afternoon. The up-regulation of TOC1 under higher ABA levels resulted in the suppression of expression of TOC1 target genes in our simulations (Additional file 1: Figure S3), which agrees with the data [3]. The model also suggests that the up-regulation of TOC1 by ABA should lengthen the circadian period $(1.3 \mathrm{~h})$ under free-running conditions (Figure $3 \mathrm{~B})$, similar to the TOC1-ox line (Figure 2). This agrees with the experimental observations, showing periods about $1.5 \mathrm{~h}$ longer than WT in the expression of the clock output gene $C A B 2$ in plants treated with high exogenous $A B A$ concentrations [29].

Altogether, the extension of the P2011 model including the widespread repression of core components by TOC1 (Figure 1A) and the inclusion of the regulatory function of ABA on TOC1 expression allowed us to improve the description of multiple datasets on the clock kinetics in wild type and mutant plants, and suggest mechanisms to explain previously paradoxical data.

\section{Gating of ABA signalling by the clock}

We next examined the biological relevance of the interactions between ABA and the circadian clock. To that end, we extended the model to the regulation of an important physiological process downstream of the clock - stomatal aperture. The equation for stomatal aperture describes the regulation of stomatal dynamics by light and by ABA signals $[10,18]$. The simulated diel kinetics of stomatal aperture are shown on Figure 4, together with data from wild-type Arabidopsis plants in the same conditions [18]. Light activation results in a more open state of stomata during the day than in the night, in agreement with the experimental data [18]. Stomatal dynamics also have a pronounced diurnal pattern related to $\mathrm{ABA}$ signalling as discussed below. Figure $5 \mathrm{~A}$ shows simulated stomatal dynamics under 

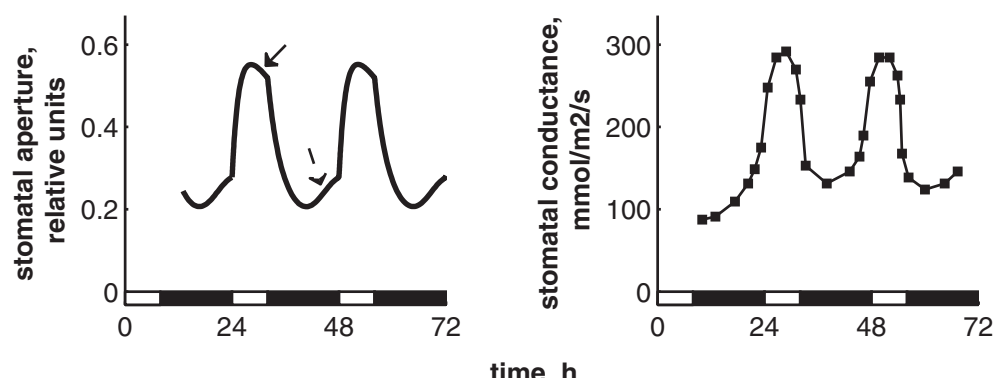

Figure 4 The kinetics of stomata opening in 8 L:16D diel cycles in the model (A) and in the experimental data (B), redrawn from [18]. Zero time corresponds to dawn. Solid and dashed arrows on A show the "dusk" and "dawn" anticipations respectively as described in the text.

different ABA levels. Increased ABA levels correspond to drought conditions, which lead to a more closed state of stomata, in agreement with the data [10]. The closing of stomata by ABA is a well-known physiological response in plants, which is important for the reduction of transpiration under drought [10,11]. Additionally to the modulation of the average daily size of the stomatal pore, ABA regulates the peak and trough phases of stomatal aperture. In the model, ABA signal is gated by the clock through $A B A R$ transcription in the morning and the following gradual increase of SnRK2 (SNF1/SucroseNonfermenting Kinase1-related protein kinase) activity during the day (Figure 1B, [11]). The SnRK2 kinase in turn regulates multiple downstream processes including the dynamics of stomatal aperture [11], as described in the Methods. Figure 5A demonstrates that the resulting diurnal timecourse of stomata aperture is characterized by a morning peak with some decline towards dusk -"dusk anticipation", resulting from higher sensitivity of stomata to ABA in the afternoon. Similarly, stomata are less sensitive to ABA before dawn, which results in an increased stomatal aperture before dawn - "dawn anticipation". Both dawn and dusk anticipations agree with the data [10] and are important for closing stomata in the heat of the afternoon to reduce a water loss and opening of stomata in the cool of the morning to prepare plants for photosynthesis after dawn [30]. The modelling here allowed us to describe the data on stomatal dynamics and showed that circadian regulation of ABAR transcription was consistent with the gating of the ABA signalling by the circadian clock and the resulting rhythmic stomatal dynamics.

The circadian regulation resulted in distinctive, freerunning rhythms of stomatal opening under constant light conditions for simulated wild type and mutant plants (Figure 5B), similar to those observed experimentally [18]. The period of this rhythmic clock output corresponded to the one of the clock itself in our simulations, in agreement with data [18]. Figure 5B demonstrates this for the shortand long-period mutants toc1 and prr7/prr9. Additionally to the change in period length, the simulated toc1 mutant had smaller stomatal aperture compared to wild type (Figure 5B). To explore the effect of TOC1 on stomata in more detail, we compared the dynamics of stomata in wild type, toc1 mutant and TOC1-ox lines. Figure 6A demonstrates that the simulated stomata were more open in the TOC1-ox and more closed in the toc1 compared to wild type, which fully agree with the experimental observations [9]. This effect was due to the direct inhibition of $A B A R$ expression by TOC1. Simulation of the model showed that this effect was especially pronounced under high

stomatal aperture, relative units
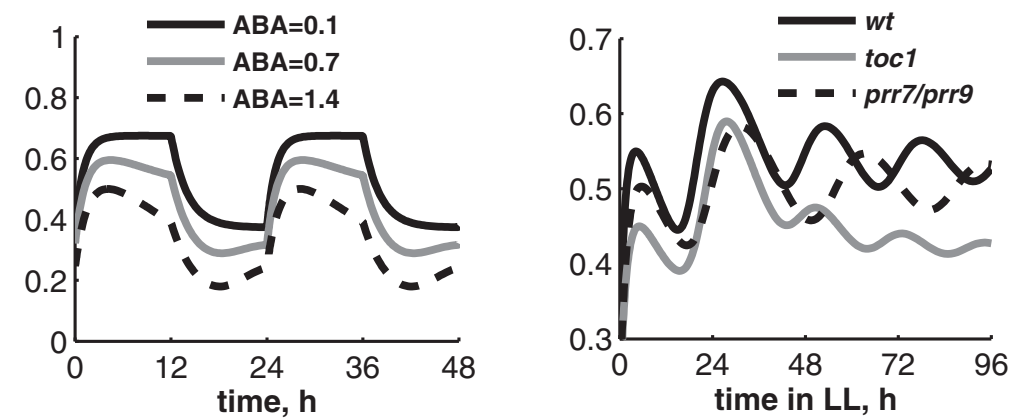

Figure 5 Simulated kinetics of stomata opening and its regulation by ABA and the circadian clock. A: Diurnal profiles of stomata aperture of wild type plants under 12 L:12D conditions for various ABA levels; B: Free running rhythms of stomata aperture under constant light conditions, calculated for wild type, toc1 and prr7/prr9 mutants. 
A

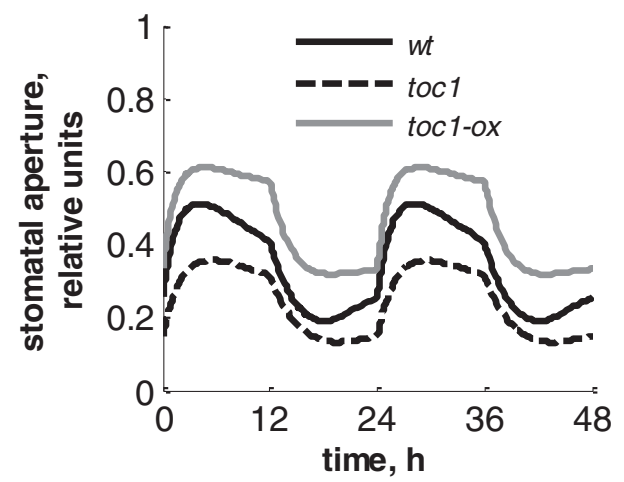

B

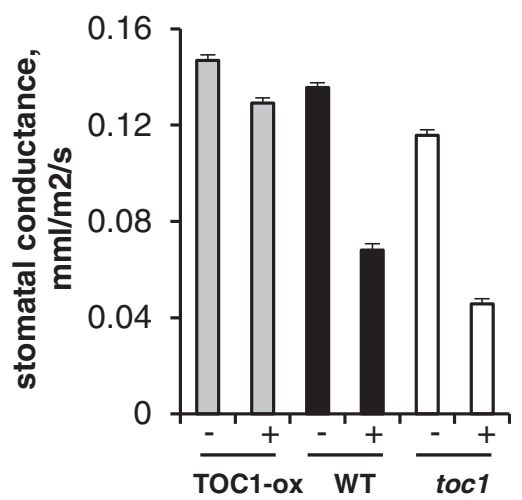

Figure 6 Effect of TOC1 on stomata size. A: Simulated time courses of stomata aperture in wild type, TOC1-ox and toc1 mutant under 12 L:12D cycle for $A B A=1.3$. TOC1-ox was simulated at $n 16=0.3$. B: Experimental data on stomata conductance in wild type, TOC1-ox and toc1 mutant in absence (-) and presence (+) of $5 \mu \mathrm{M}$ of exogenous ABA. Data are redrawn from [9].

ABA levels (Figure 6B, [9]). Additionally, the model suggests that TOC1 regulation will alter the diurnal gating of the stomatal response to $\mathrm{ABA}$, because both simulated TOC1-ox and toc1 mutant lines had less pronounced anticipation of dawn and dusk (Figure 6A).

\section{Conclusions}

The revised clock model explains clock gene profiles of mutants with altered TOC1 expression, resulting from the pervasive transcriptional repression by TOC1. The model was extended to explore the mutual connections between TOC 1 targets and ABA signalling, resulting in downregulation of clock gene expression and lengthening of the free running period under high ABA conditions. Moreover, TOC1's participation in the rhythmic gating of ABA signalling can explain well-characterised rhythms in physiological processes downstream of ABA, such as the regulation of stomata aperture. The clock effects on stress signalling are significant at the wholeplant level, as plant survival under dry conditions was reduced in TOC1-ox plants and increased in toc1 mutants [9]. Moreover, the close overlap between TOC1- and ABA-regulated genes in transcriptome profiles [9] suggests that other ABA-mediated stress responses are similarly responsive to TOC1. Integrating such physiological response pathways, even if they are represented in a simplified way, with the clock model will allow to expand our knowledge on the temporal regulation of plant physiology in its normal context, the unavoidable day-night cycle.

\section{Methods}

The system of ordinary differential equations was solved using MATLAB, integrated with the stiff solver ode15s (The MathWorks UK, Cambridge). The SBML version of the model will be available upon publication from the Biomodels database [31] (accession number BIOMD0000000445) and the Plant Systems Modelling portal (http://www.plasmo.ed.ac.uk). A MATLAB version of the model is available from the authors upon request.

\section{Additional files}

Additional file 1: Figure $\mathbf{S 1}$. The profiles of the main components of ABA signalling, simulated under 12 L:12D diel cycles. The daily changes in the levels of ABA-ABAR, active PP2C and SnRK2 are shown by black, blue and red lines, respectively. Figure $\mathbf{S 2}$. The change in the relative amplitude and period of LHY mRNA in constant light conditions measured under $10 \%$ increase and decrease of the model parameters of inhibition of target genes by TOC1 and ABA signalling to the clock. Figure S3. Relative changes in the peak expression levels of the clock genes at varied ABA values. Simulations were done under 12 L:12D conditions. Expression levels were normalized to the value in absence of ABA.

Additional file 2: Table S1. Optimal set of the model parameter values.

\section{Abbreviations}

LHY: LATE ELONGATED HYPOCOTYL; CCA1: CIRCADIAN CLOCK ASSOCIATED 1; PRR9: PRR7, PRR5, PSEUDO-RESPONSE REGULATORS 9, 7, 5; TOC1: TIMING OF CAB EXPRESSION 1; GI: GIGANTEA; ZTL: ZEITLUPE; ELF3: EARLY FLOWERING 3; ELF4: EARLY FLOWERING 4; LUX: LUX ARRHYTHMO; COP1: CONSTITUTIVE PHOTOMORPHOGENIC 1; EC: Evening complex; ABA: Abscisic acid; ABAR: ABA receptor (magnesium-chelatase $\mathrm{H}$ subunit); ABRE: ABA-responsive elements on gene promoters; PP2C: Protein phosphatase of 2C type; SnRK2: SNF1 (Sucrose-Nonfermenting Kinase1)related protein kinase; 12 L:12D: 12 h light/12 h dark daily cycle of light.

\section{Competing interests}

The authors declare that they have no competing interest.

\section{Authors' contributions}

AP, PM, AJM designed the model and drafted the manuscript. AP performed the simulations. All authors read and approved the final manuscript.

\section{Acknowledgements}

This work was supported by the European Commission FP7 Collaborative Project TiMet (project 245143). SynthSys is a Centre for Integrative and Systems Biology supported by BBSRC and EPSRC award D019621. Work in P. M. laboratory is supported by grants from the Ramón Areces Foundation, from the Spanish Ministry of Science and Innovation (MICINN) (BIO2010- 
16483) and from EUROHORCS (European Heads Of Research Councils) and the European Science Foundation (ESF) through the EURYI Award.

\section{Author details}

'School of Biological Sciences, University of Edinburgh, Mayfield Road, Edinburgh EH9 3JH, UK. ${ }^{2}$ Centre for Research in Agricultural Genomics (CRAG), Consortium CSIC-IRTA-UAB-UB, Parc de Recerca UAB, Bellaterra (Cerdanyola del Vallés) 08193, BarcelonaSpain. ${ }^{3}$ SynthSys, University of Edinburgh, C.H. Waddington Building, Mayfield Road, Edinburgh EH9 3JD, UK. Institute for Complex Systems and Mathematical Biology, University of Aberdeen, Meston Building, Aberdeen AB24 3UE, UK.

Received: 10 October 2012 Accepted: 8 March 2013

Published: 19 March 2013

\section{References}

1. Zhang EE, Kay SA: Clocks not winding down: unravelling circadian networks. Nat Rev Mol Cell Biol 2010, 11:764-776.

2. Pokhilko A, Fernandez AP, Edwards KD, Southern MM, Halliday K, Millar AJ: The clock gene circuit in Arabidopsis includes a repressilator with additional feedback loops. Mol Syst Biol 2012, 8:574-587.

3. Huang W, Perez-Garcia P, Pokhilko A, Millar AJ, Antoshechkin I, Riechmann $J$, Mas $P$ : Mapping the core of the arabidopsis circadian clock defines the network structure of the oscillator. Science 2012, 336:75-79.

4. Alabadi D, Oyama T, Yanovsky MJ, Harmon FG, Mas P, Kay SA: Reciprocal regulation between TOC1 and LHY/CCA1 within the Arabidopsis circadian clock. Science 2001, 293:880-883.

5. Pruneda-Paz JL, Breton G, Para A, Kay SA: A functional genomics approach reveals $\mathrm{CHE}$ as a component of the Arabidopsis circadian clock. Science 2009, 323:1481-1485.

6. Baudry A, Ito S, Song YH, Strait AA, Kiba T, Lu S, Henriques R, Pruneda-Paz $J \mathrm{~L}$, Chua NH, Tobin EM, et al: F-box proteins FKF1 and LKP2 act in concert with ZEITLUPE to control Arabidopsis clock progression. Plant Cell 2010, 22:606-622.

7. Gendron JM, Pruneda-Paz JL, Doherty CJ, Gross AM, Kang SE, Kay SA: Arabidopsis circadian clock protein, TOC1, is a DNA-binding transcription factor. Proc Natl Acad Sci USA 2012, 109:21152-21157.

8. Locke JC, Southern MM, Kozma-Bognar L, Hibberd V, Brown PE, Turner MS, Millar AJ: Extension of a genetic network model by iterative experimentation and mathematical analysis. Mol Syst Biol 2005, 1:0013.

9. Legnaioli T, Cuevas J, Mas P: TOC1 functions as a molecular switch connecting the circadian clock with plant responses to drought. EMBO J 2009, 28:3745-3757.

10. Correia MJ, Pereira JS, Chaves MM, Rodrigues ML, Pacheco CA: ABA xylem concentrations determine maximum daily leaf conductance of fieldgrown Vitis vinifera L. plants. Plant Cell Environ 1995, 18:511-521.

11. Raghavendra AS, Gonugunta VK, Christmann A, Grill E: ABA perception and signalling. Trends Plant Sci 2010, 15:395-401.

12. Shen YY, Wang XF, Wu FQ, Du SY, Cao Z, Shang Y, Wang XL, Peng CC, Yu $\mathrm{XC}$, Zhu SY, et al: The Mg-chelatase $\mathrm{H}$ subunit is an abscisic acid receptor. Nature 2006, 443:823-826.

13. Wu FQ, Xin Q, Cao Z, Liu ZQ, Du SY, Mei C, Zhao CX, Wang XF, Shang Y, Jiang $\mathrm{T}$, et al: The magnesium-chelatase $\mathrm{H}$ subunit binds abscisic acid and functions in abscisic acid signaling: new evidence in Arabidopsis. Plant Physiol 2009, 150:1940-1954.

14. Adams R, Clark A, Yamaguchi A, Hanlon N, Tsorman N, Ali S, Lebedeva G, Goltsov A, Sorokin A, Akman OE, et al: SBSI: an extensible distributed software infrastructure for parameter estimation in systems biology. Bioinformatics 2013, 29:664-665.

15. Pokhilko A, Ramos JA, Holtan H, Maszle DR, Khanna R, Millar AJ: Ubiquitin ligase switch in plant photomorphogenesis: A hypothesis. $J$ Theor Biol 2011, 270:31-41.

16. Neales TF, Masia A, Zhang J, Davies WJ: The effects of partially drying part of the root system of helianthus annuus on the abscisic acid content of the roots, xylem sap and leaves. J Exp Botan 1989, 40:1113-1120.

17. Harmer SL, Kay SA: Positive and negative factors confer phase-specific circadian regulation of transcription in Arabidopsis. Plant Cell 2005, 17:1926-1940.

18. Dodd AN, Parkinson K, Webb AAR: Independent circadian regulation of assimilation and stomatal conductance in the ztl-1 mutant of Arabidopsis. New Phytol 2004, 162:63-70.
19. Farre EM, Harmer SL, Harmon FG, Yanovsky MJ, Kay SA: Overlapping and distinct roles of PRR7 and PRR9 in the Arabidopsis circadian clock. Curr Biol 2005, 15:47-54.

20. Mas P, Kim WY, Somers DE, Kay SA: Targeted degradation of TOC1 by ZTL modulates circadian function in Arabidopsis thaliana. Nature 2003, 426:567-570

21. Mas P, Alabadi D, Yanovsky MJ, Oyama T, Kay SA: Dual role of TOC1 in the control of circadian and photomorphogenic responses in Arabidopsis. Plant Cell 2003, 15:223-236.

22. Strayer C, Oyama T, Schultz TF, Raman R, Somers DE, Mas P, Panda S, Kreps JA, Kay SA: Cloning of the Arabidopsis clock gene TOC1, an autoregulatory response regulator homolog. Science 2000, 289:768-771.

23. McWatters HG, Bastow RM, Hall A, Millar AJ: The ELF3 zeitnehmer regulates light signalling to the circadian clock. Nature 2000, 408:716-720.

24. McWatters HG, Kolmos E, Hall A, Doyle MR, Amasino RM, Gyula P, Nagy F, Millar AJ, Davis SJ: ELF4 is required for oscillatory properties of the circadian clock. Plant Physiol 2007, 144:391-401.

25. Hazen SP, Schultz TF, Pruneda-Paz JL, Borevitz JO, Ecker JR, Kay SA: LUX ARRHYTHMO encodes a Myb domain protein essential for circadian rhythms. Proc Natl Acad Sci USA 2005, 102:10387-10392.

26. Martin-Tryon EL, Kreps JA, Harmer SL: GIGANTEA acts in blue light signaling and has biochemically separable roles in circadian clock and flowering time regulation. Plant Physiol 2007, 143:473-486.

27. Makino S, Matsushika A, Kojima M, Yamashino T, Mizuno T: The APRR1/ TOC1 quintet implicated in circadian rhythms of Arabidopsis thaliana: I. Characterization with APRR1-overexpressing plants. Plant Cell Physiol 2002, 43:58-69.

28. Ding Z, Doyle MR, Amasino RM, Davis SJ: A complex genetic interaction between Arabidopsis thaliana TOC1 and CCA1/LHY in driving the circadian clock and in output regulation. Genetics 2007, 176:1501-1510

29. Hanano S, Domagalska MA, Nagy F, Davis SJ: Multiple phytohormones influence distinct parameters of the plant circadian clock. Genes Cells 2006, 11:1381-1392

30. Webb AAR: Stomatal rhythms. In Biological rhythms and photoperiodism in plants. Edited by Lumsden PJ, Millar AJ. Oxford: Bios Scientific Publications; 1998:66-79.

31. Le Novere N, Bornstein B, Broicher A, Courtot M, Donizelli M, Dharuri H, Li L, Sauro H, Schilstra M, Shapiro B, et al: BioModels Database: a free, centralized database of curated, published, quantitative kinetic models of biochemical and cellular systems. Nucleic Acids Res 2006, 34:D689-691.

doi:10.1186/1752-0509-7-23

Cite this article as: Pokhilko et al: Modelling the widespread effects of TOC1 signalling on the plant circadian clock and its outputs. BMC Systems Biology 2013 7:23.

\section{Submit your next manuscript to BioMed Central and take full advantage of:}

- Convenient online submission

- Thorough peer review

- No space constraints or color figure charges

- Immediate publication on acceptance

- Inclusion in PubMed, CAS, Scopus and Google Scholar

- Research which is freely available for redistribution 